\title{
MOTOR DE RELUCTANCIA CONMUTADA: MODELADO, SIMULACIÓN Y CONTROL
}

Eduard Galvis', Virgilio Vásquez², Julián Ferreira³

\section{RESUMEN}

El motor de reluctancia conmutada (SRM) es una máquina eléctrica con polos dobles salientes. Lo anterior significa que tiene polos salientes tanto en el estator como en el rotor. La construcción de un SRM es simple comparada con otro tipo de máquinas eléctricas. El rotor no es de un material magnético y tampoco se encuentra embobinado; las bobinas se encuentran únicamente en el estator. En este trabajo se presenta una estrategia de control de la velocidad angular para este tipo de motores. Se emplea el software MATLAB/Simulink para describir el modelo no lineal y su respectiva simulación digital. El control de velocidad angular del motor se logra empleando la perspectiva de control por corriente utilizando un controlador proporcional-integral, $\mathrm{Pl}$.

Palabras Claves: Matlab/Simulink, Control PI, Motor de reluctancia conmutada

\section{ABSTRACT}

The switched reluctance motor (SRM) is a doubly-salient machine. This means that it has salient poles on both the rotor and stator. A SRM construction is simple as compared to other types of electric motors. The rotor has no windings or magnet; the windings are concentrated in the stator. The purpose of this article is the development of a speed control strategy for this kind of motor. Description of a nonlinear SRM model and its respective simulation in Matlab/Simulink environment is presented. The constant rotor speed control of SRM is achieved from current control perspective with a PI controller.

Keywords: Matlab/Simulink, PI Controller, Switched reluctance motor

\section{INTRODUCCION}

Una máquina de reluctancia, es una máquina eléctrica en la cual el par es producido por la tendencia del rotor a moverse a una posición donde la inductancia del embobinado excitado es maximizada. Las máquinas de reluctancia pueden ser sincrónicas o conmutadas. El Motor de Reluctancia Conmutada (SRM), también conocido como máquina de reluctancia variable (VRM) es una máquina rotativa que no utiliza escobillas ni imanes permanentes. Dentro de las características destacables de este tipo de motor se encuentran:

Simplicidad constructiva comparada con otros tipos de motores eléctricos, lo cual disminuye los costos de fabricación.

- Elevado rendimiento (eficiencia mayor a 90\% típicamente)

Producen alto par a bajas velocidades.

La corriente de rotor no necesita ser bidireccional, lo cual simplifica el diseño del circuito de control y potencia.

- Es una máquina sin escobillas, lo que facilita su mantenimiento.

Prestaciones con baja dependencia de la temperatura.

Dentro de las limitaciones de estos motores se destacan:

A diferencia de otras máquinas eléctricas convencionales, no pueden operar directamente con una fuente CA o CD. Es obligatorio el empleo de un controlador electrónico.

- La característica del par exhibe un alto rizado comparado con otras máquinas eléctricas. El rizado son componentes de alta frecuencia que aparecen en las formas de onda de las variables del motor, como el par, corrientes de fase.

\footnotetext{
1 egalvis@bari.ufps.edu.co. Estudiante MCl. ITESM-CEM. México. Integrante GIAC - UFPS,

2 vlopez@itesm.mx. Profesor Investigador. Dpto. Mecatrónica. ITESM-CEM. México,

3 jfereir@bari.ufps.edu.co Profesor Dpto. Electricidad y Electrónica. UFPS. Integrante GIAC - UFPS.
} 


\section{MOTOR DE RELUCTANCIA CONMUTADA: MODELADO, SIMULACIÓN Y CONTROL}

A manera de antecedentes, en la referencia [1] se muestra un análisis del modelo matemático limitado a la región lineal de la característica magnética, en la referencia [2] se realiza el análisis del comportamiento del motor en la región saturada y se describen características de control de velocidad angular, con dos variables de control: amplitud de la corriente (ó ángulo de encendido) y ángulo de apagado.

En la referencia [7] se plantea un modelo no lineal que relaciona las variables más importantes en el diseño de una máquina de reluctancia variable, para llegar una expresión analítica de la característica de magnetización. Se realiza además de una verificación experimental con tres modelos de SRM de diferentes tamaños y estructuras. La comparación entre los datos de magnetización medidos experimentalmente con los datos calculados del modelo analítico reflejaron una buena aproximación.

Posteriormente la referencia [4] toma los resultados de [7] y desarrolla un modelo no lineal versátil el cual se ha implementado como un bloque simPowersystems en el ambiente Matlab/Simulink para 3 tipos de SRM. Concluyen que los resultados obtenidos con el modelo son lo suficientemente exactos para ser empleados en el desarrollo y evaluación de algoritmos de control para este tipo de motores. En este mismo artículo se muestran dos modos llamados de corriente controlada y de voltaje alimentado para hacer funcionar el motor mediante la activación sucesiva de las fases del estator; sin embargo no se realiza ningún control específico de velocidad, posición o par.

En el presente trabajo se toman los resultados de la referencia [4], a partir las expresiones analíticas del flujo ligado y par eléctrico se realiza una simulación del modelo del motor. Además se plantea el control de la velocidad angular mediante el empleo de un controlador Proporcional-Integral.

\section{EL SRM}

El SRM es topológica y electromagneticamente similar a un motor a pasos. Al no tener imanes o embobinados en el rotor se simplifica su construcción [5]. Con la simplicidad se eliminan algunos problemas comunes en las máquinas eléctricas como lo son la magnetización y desmagnetización en el entrehierro. Para alcanzar desempeños competitivos se hace necesario controlar electrónicamente la forma de onda de corriente. Se debe incluir retroalimentación de la posición del rotor y sincronizar la conmutación de las corrientes de fase con la posición del rotor de forma adecuada.

En relación a su construcción y requerimientos del controlador, un SRM es muy diferente de los motores CD y CA. Todas las características relacionadas con la máquina (flujo ligado, inductancia, par de fase, etc.) son funciones no lineales de la posición del rotor y la corriente de fase. A esto se adiciona la operación intencional en la región de saturación de la curva de magnetización, con el objeto de maximizar la relación par a masa. Lo anterior torna compleja la descripción analítica del comportamiento del motor. Las no linealidades llevan a altos rizados en el par cuando la excitación de las bobinas se hace con pulsos rectangulares.

Para su funcionamiento en el estator se colocan bobinas, que conectadas a pares diametralmente opuestos formando las fases del motor. La excitación de una de estas fases genera un campo magnético que se cierra através del rotor, el cual para alcanzar la posición en donde la reluctancia del circuito sea mínima, se alineará con los polos del estator, por lo tanto se genera un par. La figura 1 muestra la sección transversal de dos motores de reluctancia conmutada, el motor de la izquierda con 6 polos en el estator y 4 polos en el rotor (6/4) y el otro con 8 polos en el estator y 6 polos en el rotor (8/6). Los ángulos $\beta$ s y $\beta$ r con vértice en el centro del rotor, describen los arcos con la longitud de polos en el estator y el rotor respectivamente. Si los 
polos del estator están enfrentados a dos polos del rotor (alineados), será una posición de mínima reluctancia y máxima inductancia. El rotor se puede hacer rotar en cualquier dirección energizando secuencialmente las fases consecutivas del estator [3].

Figura 1. Dos estructuras de SRMs: 6/4 (izquierda) y $8 / 6$ (derecha)
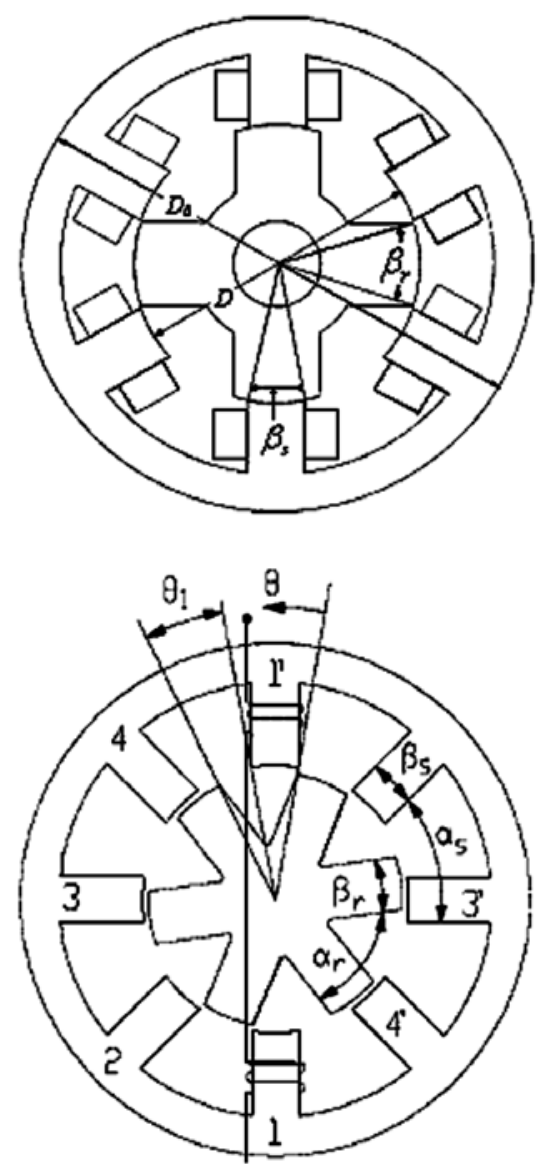

\section{MODELO MATEMÁTICO DE UN SRM}

Los modelos matemáticos de los SRM generalmente están conformados por tres partes: el modelo eléctrico, la característica del torque y el modelo mecánico [8]. La configuración en bloques de un modelo puede verse en la figura 2 , donde las señales de entrada son los voltajes de estator y las variables de salida son de tipo mecánico.
Figura 2. Configuración de un modelo equivalente de un SRM.

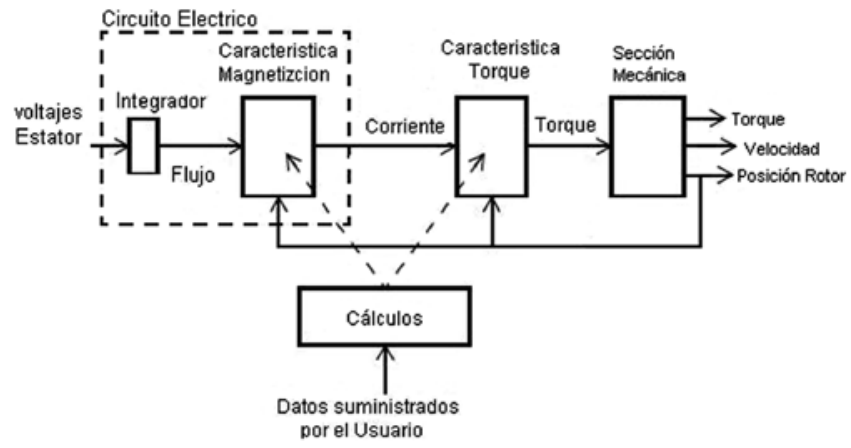

El circuito eléctrico para una sola fase de estator se puede modelar como una red $R L$ con la fuente de excitación $v(t)$ como se muestra en la figura 3 .

Figura 3. Circuito equivalente para una fase del motor

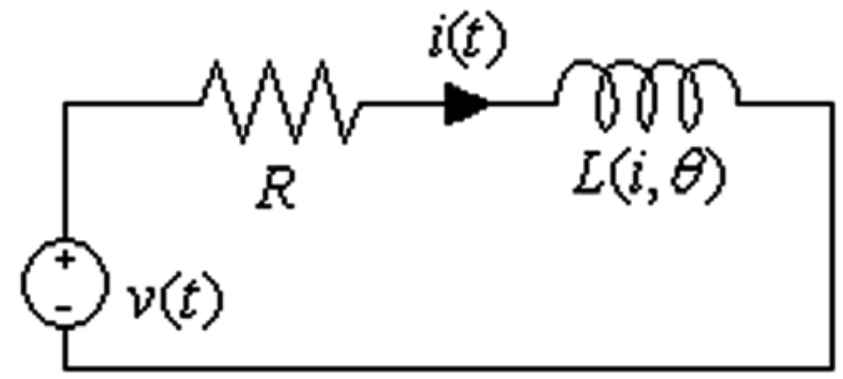

Si se aplica la ley de voltajes de Kirchoff (se omite la dependencia del tiempo por simplicidad)

$$
v=i R+\frac{d \lambda(\theta, i)}{d t}
$$

Donde $\theta$ es la posición del rotor, $R$ es la resistencia de fase (se asume constante por simplicidad). es el flujo magnético. El acoplamiento mutuo entre fases es normalmente cero o muy pequeño, por ello se ignora. La derivada temporal del flujo se puede escribir como la suma de dos derivadas parciales, entonces la ecuación (1) se puede reescribir:

$$
\frac{d i}{d t}=\frac{1}{L(\theta, i)}\left(v-i R-\omega i \frac{d L(\theta, i)}{d \theta}\right)
$$

Donde $\omega=d \theta / d t$ es la velocidad angular. $L(\theta, i)=d \lambda(\theta, i) / d i$ es la inductancia instantánea. 


\section{MOTOR DE RELUCTANCIA CONMUTADA: MODELADO, SIMULACIÓN Y CONTROL}

Para un sistema electromecánico no lineal como el SRM, el par eléctrico $T(i, \theta)$, puede ser calculado por la derivada espacial de la co-energía de la máquina con respecto a la posición angular:

$$
T(i, \theta)=\frac{\partial}{\partial \theta} \int_{0}^{i} \lambda(i, \theta) \cdot d i
$$

El par total desarrollado por el motor es la suma de los pares generados por cada una de las fases:

$$
T_{e}(i, \theta)=\sum_{p=1}^{N p} T_{p}(i, \theta)
$$

Donde $N p$ es el número de pases del motor $T_{e}(i, \theta)$ es el par eléctrico total y $T_{p}$ es el par de fase.

Para propósitos de simulación, el modelo se puede implementar con dos tablas de búsqueda que calculen $i=i(\lambda, \theta)$ y $T=T(i, \theta)$, ambas basadas en la característica de magnetización del motor. En la figura 4 se muestran las curvas de magnetización de un SRM 6/4 de 64KW, empleando los datos experimentales dados en la referencia [7]. Donde se han parametrizado las relaciones de flujo-corriente con la posición del rotor.

Figura 4. Curvas de Magnetización Experimental de un SRM

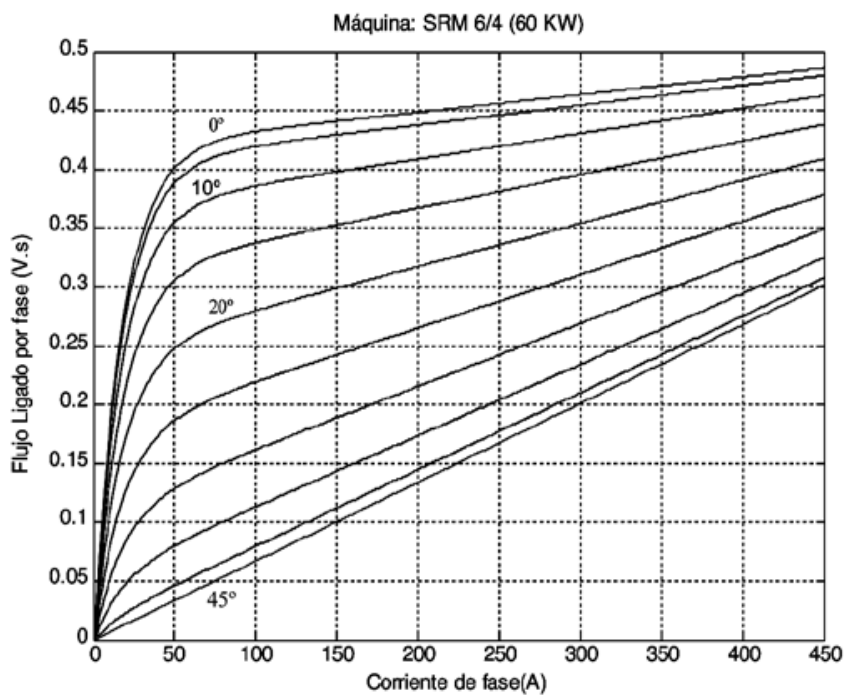

En la referencia [4] se calculan las expresiones ana- líticas para el flujo ligado y el par usando análisis de ajuste de curvas a partir de los principales parámetros normalmente disponibles o de fácil medición en el motor. La curva de magnetización se puede expresar analíticamente como una función de la corriente de estator y la posición del rotor [4]:

Donde:

$$
\begin{gathered}
\lambda(i, \theta)=L_{q} i+\left[L_{d s a t} i+A\left(1-e^{-B i}\right)-L_{q} i\right] f(\theta) \\
f(\theta)=\left(\frac{2 N_{r}^{3}}{\pi^{3}}\right) \theta^{3}-\left(\frac{3 N_{r}^{2}}{\pi^{2}}\right) \theta^{2}+1
\end{gathered}
$$

$L_{q}$ es la inductancia mínima. $L_{d s a t}$ es la inductancia saturada. $A$ y $B$ son constantes determinadas por las condiciones en $i=0$ e $i=I m$. Donde $I m$ es la corriente máxima. Suponiendo que la máquina está realmente saturada en $i=I m$ se tiene $i=\operatorname{Im}$ se tiene $e^{-B I_{m}} \approx 0$ y las constantes $A$ y $B$ se puede deducir como:

$$
A=\lambda_{m}-L_{d s a t} I_{m} \quad B=\frac{L_{d}-L_{d s a t}}{\lambda_{m}-L_{d s a t} I_{m}}
$$

Si se reemplaza la ecuación (5) en (3), se llega a una expresión más explicita del par:

$T_{e}(i, \theta)=i+\left[\frac{\left(L_{d s a t}-L_{q}\right) i^{2}}{2}+A i+\frac{A\left(1-e^{-B i}\right)}{B}\right] f^{\prime}(\theta)$

Donde $\quad f^{\prime}(\theta)=\left(\frac{6 N_{r}^{3}}{\pi^{3}}\right) \theta^{2}-\left(\frac{6 N_{r}^{2}}{\pi^{2}}\right) \theta$

La dinámica del modelo mecánico puede ser descrita por [4]:

$$
\begin{gathered}
\omega=\frac{d \theta}{d t} \\
J \frac{d \omega}{d t}=T_{e}(\theta, i)-T_{m}(\theta, \omega)
\end{gathered}
$$

Donde $J$ es la inercia total (motor + carga). $T_{m}(\theta, \omega)$ es el par mecánico, este se puede escribir sin pérdida de generalidad como:

$$
T_{m}(\theta, \omega)=b \omega+T_{L}
$$

Donde $b$ es la fricción viscosa y $T_{L}$ el par de carga. Si se aplica transformada de Laplace con condiciones iniciales iguales a cero, la ecuación se convierte en: 


\section{MOTOR DE RELUCTANCIA CONMUTADA: MODELADO, SIMULACIÓN Y CONTROL}

$$
\omega(s)=\frac{T_{e}(s)-T_{L}(s)}{s J+b}
$$

En resumen las ecuaciones (2), (9) y (10) representan el modelo dinámico del SRM.

\section{SIMULACIÓN EN MATLAB/SIMULINK}

La simulación se hace a partir de las características de magnetización (flujo ligado como una función de la corriente y la posición del rotor). El modelo matemático de un SRM de tres fases, con 4 polos en el rotor, 6 polos en estator (SRM 6/4) se ha implementado en Simulink (figura 5).

Figura 5. Modelo en Bloques de SRM 6/4.

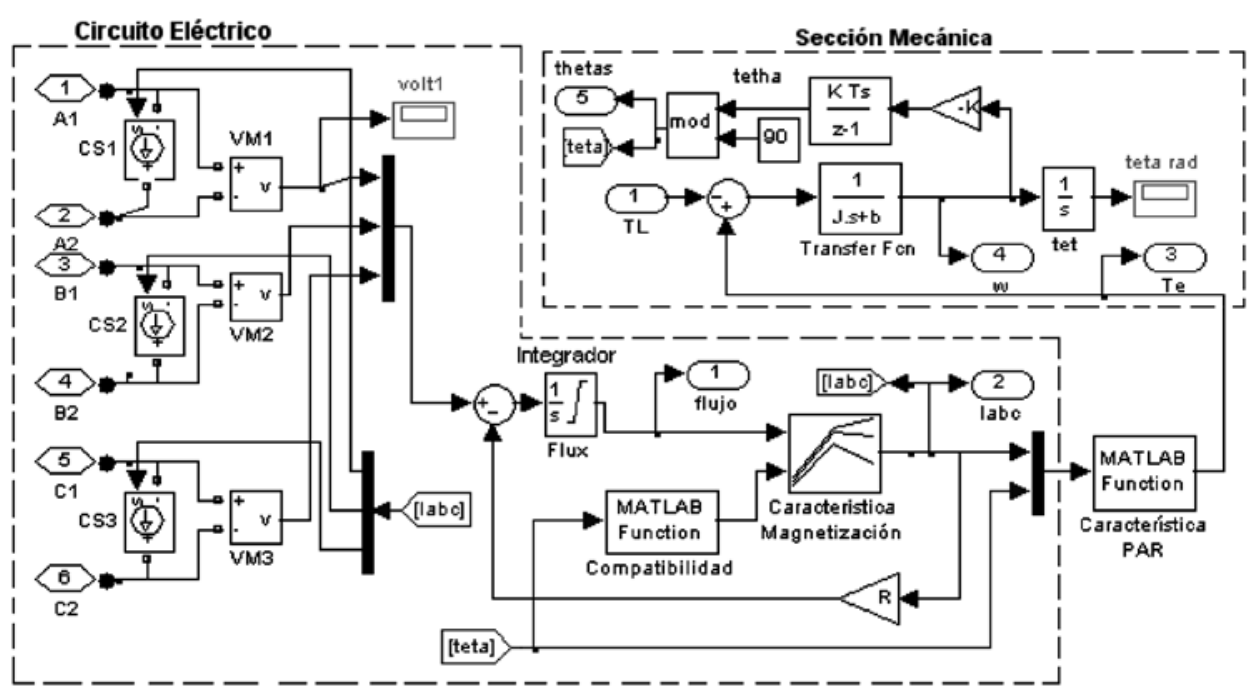
en la tabla.

Los medidores de voltaje (VMs) se han obtenido de la librería SimPowerSystem y se emplean para procesar los voltajes de fase que actúan como entradas al sistema. El integrador del flujo se ha limitado al valor en saturación $\left(\lambda_{\max }\right)$. Las fuentes controladas de corriente CS1,CS2 y CS3 inyectan en las bobinas del estator la corriente producida por la función no lineal $i=i(\lambda, \theta)$, la cual se implementó en forma de tabla de búsqueda (Lookup table). La función de matlab llamada compatibilidad sirve como interface para adecuar los valores de los ángulos a los requeridos

El bloque funcional Característica PAR realiza la sumatoria de cada una de los torques de fase, $T_{e}=f(i, \theta)$ en

(4) después de evaluar (7). Al integrador de tiempo discreto empleado para el cálculo de $\theta$ a partir de $\omega$ se le asigna un vector con valores iniciales de $[0,-30,-60]$, es decir se considera que al inicio de la simulación una de las fases del estator (2 polos diametralmente opuestos) está enfrentada con dos polos del rotor.

Para el manejador (driver) del motor se ha empleado una topología típica de inversor con dos tran-

Figura 6. Configuración del Inversor

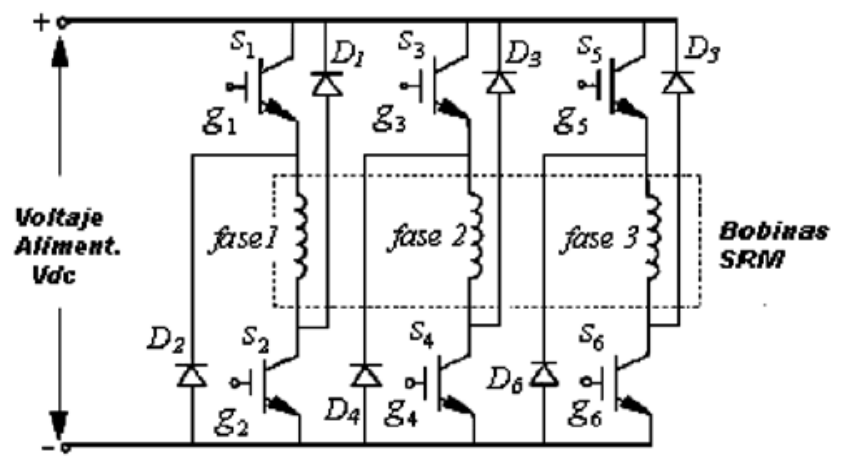

sistores y dos diodos por fase, como la mostrada en la figura 6. Las inductancias de fase están en serie con los dispositivos de conmutación.

\section{CONTROL DE VELOCIDAD DEL SRM}

En la referencia [4] se propusieron dos modos de operación de un SRM, de estos resultados se infiere que al hacer un seguimiento a una corriente de referencia constante se puede lograr que la velocidad angular 


\section{MOTOR DE RELUCTANCIA CONMUTADA: MODELADO, SIMULACIÓN Y CONTROL}

del motor se estabilice en un punto específico. Estos modos de operación son esquemas de control del tipo ON-OFF y tienen el problema de ancho de la banda diferencial del bucle de histéresis muy alto, lo cual se traduce en un rizado de magnitud considerable. En el presente trabajo se presenta una versión mejorada del enfoque de alimentación de corriente, al implementar un modulo con un controlador proporcional integrar (PI), bajo un esquema de control maestro esclavo, como se muestra en la figura 7.

Figura 7. Estructura básica del controlador se emplea la siguiente ecuación:

$$
I_{r e f}(t)=K_{p} e(t)+K i \int_{0}^{t} e(\tau) d(\tau)
$$

Donde $K_{p}$ es la ganancia proporcional, $K_{i}$ es la ganancia integral. Es común reescribir esta ecuación de la forma siguiente:

$I_{r e f}(t)=K\left(e(t)+\frac{1}{T i} \int_{0}^{t} e(\tau) d(\tau)\right)$

Donde $K=K_{p}$ y $T_{i}=K / K_{i}$ y es el tiempo integral.

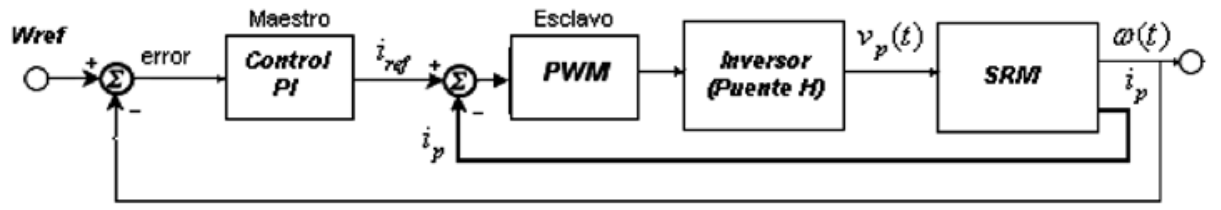

La implementación del sistema completo se muestra en la figura 8 , se emplea un modulador por ancho de pulso PWM que actúa como controlador de corriente del mo-

Para propósitos de diseño, se desea configurar un controlador para un SRM de 6/4 para el control de la velocidad angular del motor, con los siguientes objetivos:

- Estabilidad del sistema retroalimentado.

- Error en estado estacionario debido a una entrada escalón de magnitud $\omega_{\text {ref }}=1600 \mathrm{rpm}$, $e_{s s}<0.3 \mathrm{r} / \mathrm{s}(2.86 \mathrm{rpm})$

- Tiempo de establecimiento, $t_{s}<0.5 \mathrm{seg}$.

- Robustez en presencia de variaciones del par de carga.

En lazo de retroalimentación el error se puede emplear para controlar la magnitud de la corriente de referencia. Cuando el par total instantáneo es mayor que la suma de la fuerza de fricción y el par de carga $T_{L^{\prime}}$ la velocidad angular se puede controlar. De esta manera para el controlador tor siguiendo a una corriente de referencia, $i_{\text {ref }}$ dentro de una brecha diferencial o banda de histéresis. Si la corriente excede el límite superior de la banda de

Figura 8. Diagrama en Bloques del sistema completo.

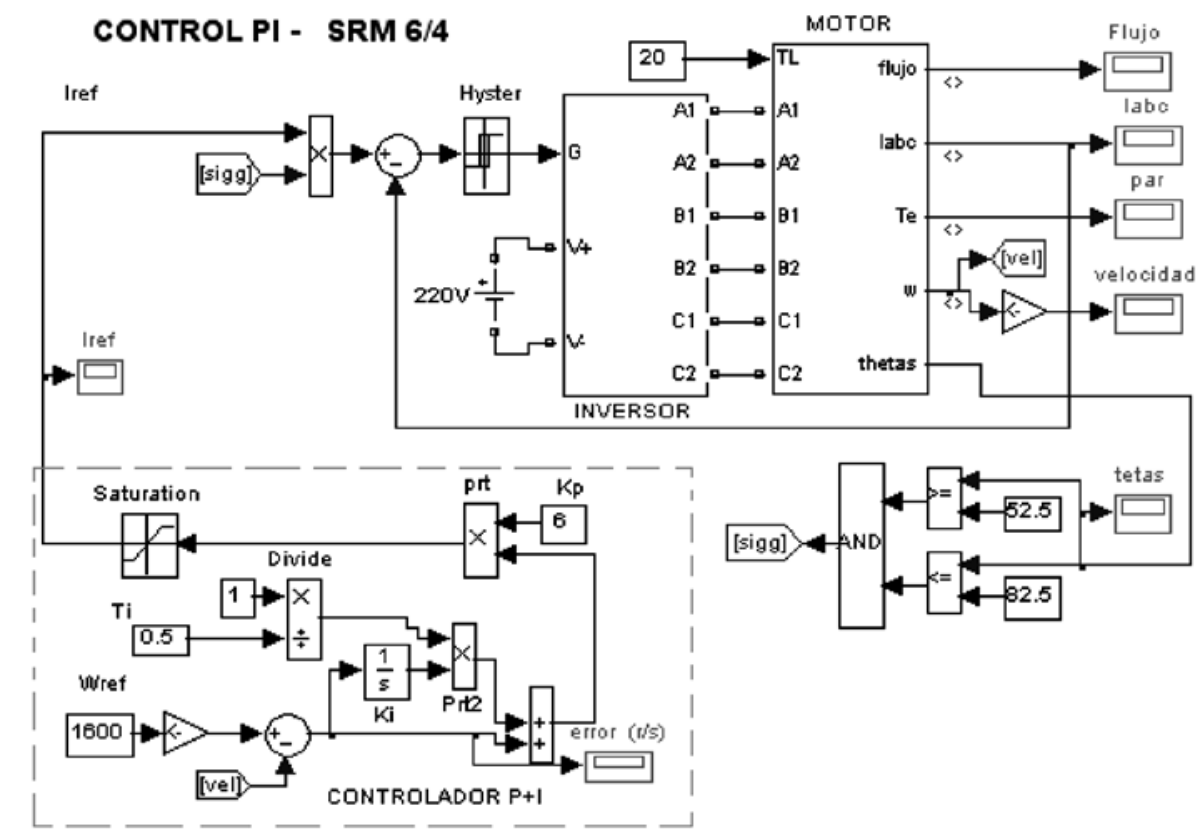




\section{MOTOR DE RELUCTANCIA CONMUTADA: MODELADO, SIMULACIÓN Y CONTROL}

histéresis, el conmutador respectivo del inversor se apagará, y consecuentemente la corriente empezará a decaer. Si la corriente es inferior que el límite inferior de la banda de histéresis, el voltaje presente en la bobina de fase del motor será $V d c$, como resultado la corriente volverá a estar dentro de la brecha diferencial.

Las gráficas obtenidas de la simulación se muestran en las figuras 9 .

Figura 9. Formas de onda para $i, i_{\text {ref }}, \lambda y T e$

El comportamiento dinámico del SRM, presenta ahora un rizado mucho menor que el propuesto en el control por corriente básico. En la figura 10 se muestra la comparación, la grafica de la derecha se ha obtenido con el control por corriente básico planteado en [4] con una $i_{\text {ref }}=200 \mathrm{~A}$, la grafica de la derecha se obtuvo con el control PI propuesto en este articulo.

Flujo Ligado (V.s)
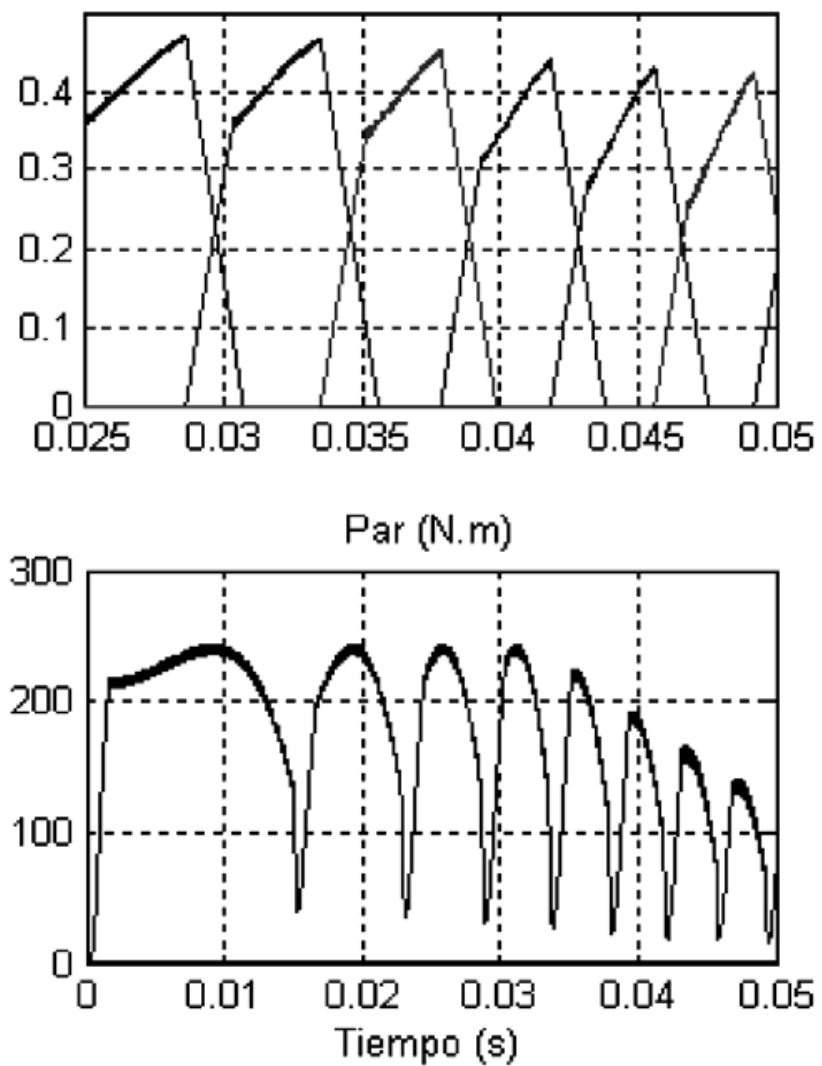
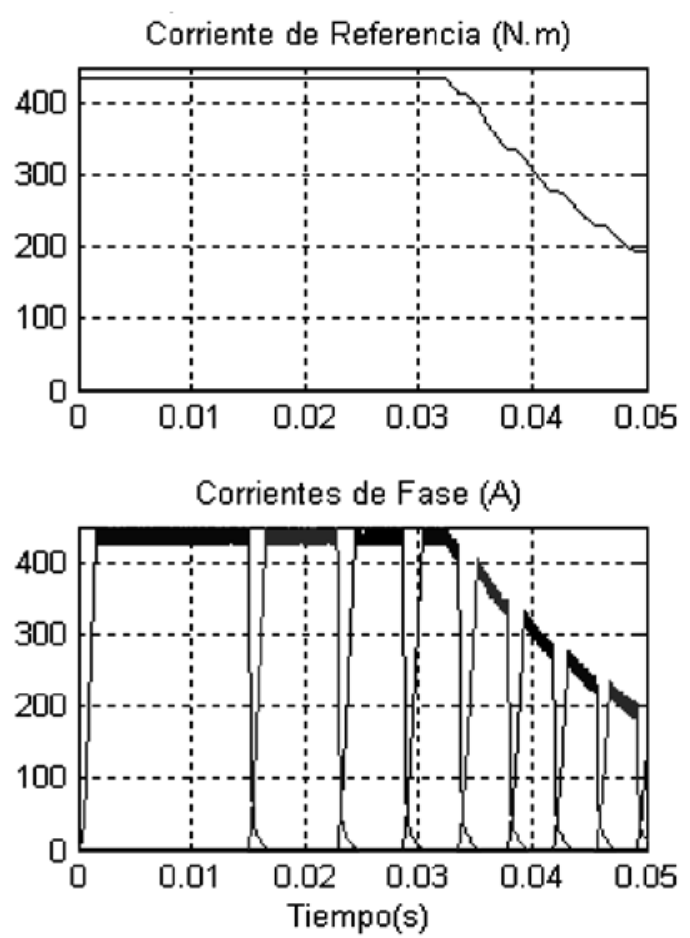

Figura 10. Corrientes de fase para control por corriente básico (arriba) y control PI (abajo).
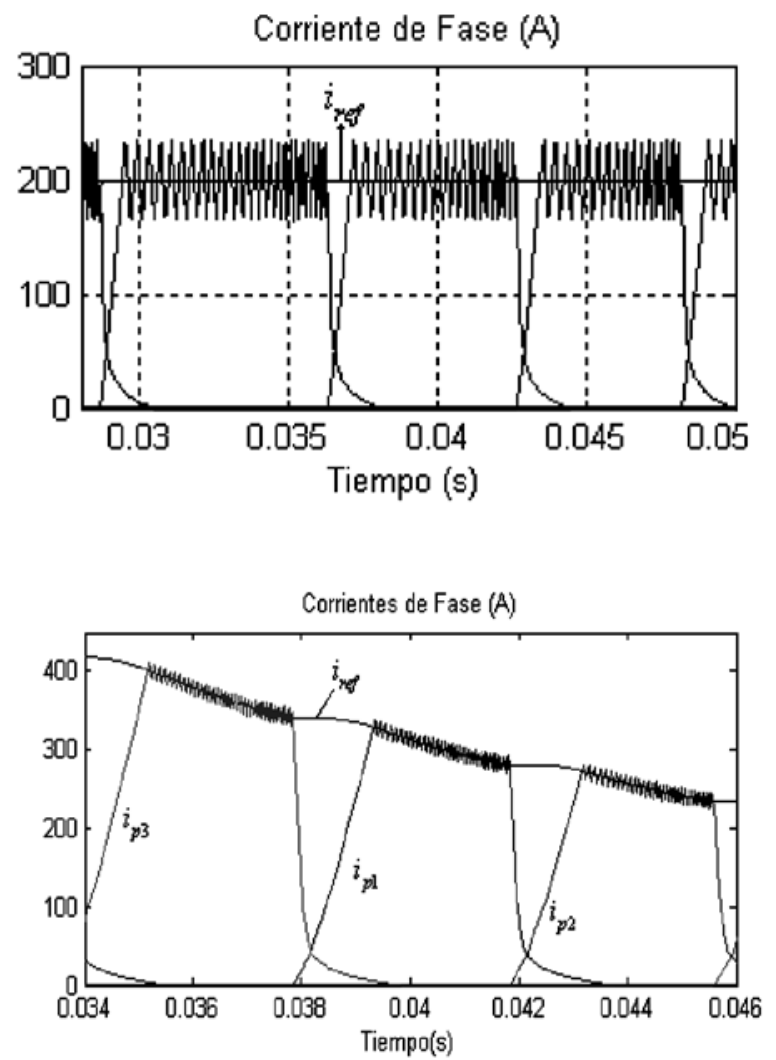


\section{MOTOR DE RELUCTANCIA CONMUTADA: MODELADO, SIMULACIÓN Y CONTROL}

Para la sintonización de las ganancias del controlador, se hacen pruebas variando la ganancia proporcional, $K_{p}$, considerando el par de la carga $T_{L}=0$. La figura 11 muestra la forma de onda de la velocidad angular para diferentes valores de ganancias del controlador. Como entrada de referencia se ha tomado un escalón de magnitud $\omega_{\text {ref }}=1600 \mathrm{rpm}$. Mientras se mantiene constante el valor del tiempo integral $T_{i}$, en un valor que permita alcanzar el tiempo de establecimiento sugerido $\left(t_{s} \leq 0.5 \mathrm{~s}\right)$.

Figura 11. Formas de onda de velocidad angular para diferentes ganancias.

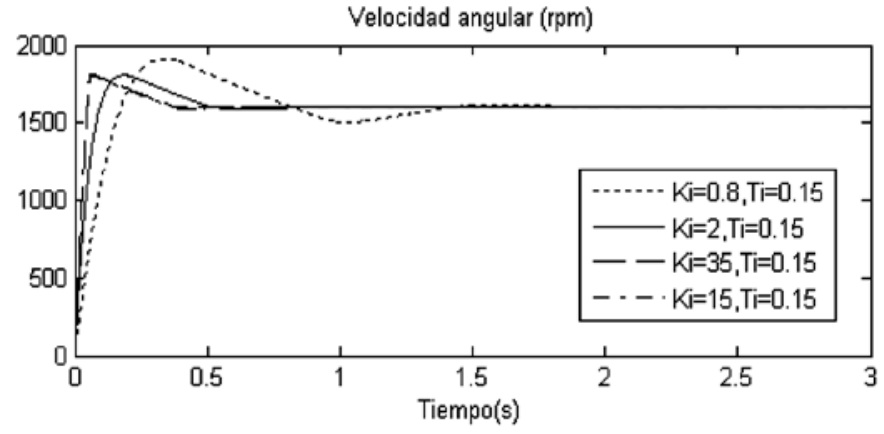

Existe un compromiso entre el porcentaje de sobreelongación máxima y el tiempo de establecimiento. Se han elegido, $K_{p}=15$ y $T_{i}=0.15$, pues permiten alcanzar los requisitos inicialmente planteados.

Se ha empleado un criterio comparativo para cuantificar el desempeño del controlador, denominado error cuadrático integral

$$
\|e\|_{2}=\left[\int_{0}^{T}\left(\omega_{r e f}-\omega(t)\right)^{2} d t\right]^{1 / 2}
$$

En este trabajo se han hecho 4 mediciones para encontrar numéricamente $\|e\|_{2}$. En el primer caso se consideran los valores nominales supuestos de los parámetros. ( $T_{L}=20 \mathrm{~N} . \mathrm{m}, R=0.05 \Omega$ ). En el segundo experimento se realiza una variación del par de carga, con una elevación de $40 \%$ en un intervalo de tiempo de $0.35 \mathrm{~s}$. Como periodo de evaluación se ha tomado un segundo.
Tabla 2. Cuantificación del error.

\begin{tabular}{|c|c|c|l|}
\hline Exp. & Parámetros & Porcentaje. incertidumbre & $\|e\|_{2}$ \\
\hline 0 & $T_{L}=20, R_{S}=0.05$ & - & 4.9476 \\
\hline 1 & Vrs. Nominales & $0 \%$ & 5.5619 \\
\hline 2 & Resist. Estator $R_{S}$ & $+30 \%$ & 5.5636 \\
\hline 3 & Par de carga $T_{L}$ & $+200 \%(0.05 ? \mathrm{t} ? 0.07 \mathrm{seg})$. & 6.2429 \\
\hline 4 & $T_{L}$ y $R_{S}$ & $+30 \% \mathrm{y}+40 \%$ & 6.2457 \\
\hline
\end{tabular}

\section{CONCLUSIONES}

El artículo ha presentado un controlador proporcional - integral en configuración de maestro-esclavo con un modulador de histéresis para el control de velocidad angular de un SRM.

Las características del par y los subsistemas eléctrico y mecánico descritos analíticamente en las referencias [4] y [7] se han simulado en el ambiente Matlab/Simulink. Se dejan sentadas las bases para posteriores trabajos acerca del tema, se ha construido y documentado de manera detallada cada uno de los bloques que conforman un SRM y los resultados de las formas de onda demostraron ser coherentes con la bibliografía revisada.

Actualmente se está trabajando en elaboración de una ley de control no lineal de estructura variable, que permita reducir aun más el rizado y por lo tanto los componentes armónicos de las señales aquí mostradas. La implementación en un motor real así como el control de posición angular y la auto-sintonización de las ganancias del controlador se consideran parte de una investigación posterior sobre el esquema maestro-esclavo propuesto en este trabajo.

\section{APENDICE}

\section{A. Parámetros de Simulación}

Método de Integración de paso fijo con fórmula de Runge Kutta de orden 4.

Tiempo de muestreo $=1 \times 10^{-6} \mathrm{~S}$ 


\section{MOTOR DE RELUCTANCIA CONMUTADA: MODELADO, SIMULACIÓN Y CONTROL}

Tabla 1: Datos del Motor SRM 6/4 de 64 KW

\begin{tabular}{|l|l|l|}
\hline & \multicolumn{1}{|c|}{ Parámetro } & \multicolumn{1}{c|}{ Valor } \\
\hline$R s$ & Resistencia por fase del Estator & $0.05 \Omega$ \\
\hline$b$ & Coeficiente de fricción viscosa & $0.02 \mathrm{~N} . \mathrm{m} . \mathrm{s}$ \\
\hline$J$ & Inercia total del motor & $0.05 \mathrm{kgm}{ }^{2}$ \\
\hline$m$ & Número de fases & 3 \\
\hline$N r$ & Número de polos & 4 \\
\hline$L_{q}$ & Inductancia desalineada de fase & $0.673 e-H$ \\
\hline$L_{d}$ & Inductancia alineada de fase & $23.63 e-H$ \\
\hline$L_{d s a t}$ & Ind. alineada en saturación & $0.153 e-H$ \\
\hline$I_{m}$ & Corriente máxima & $450 \mathrm{~A}$. \\
\hline$\lambda_{\max }$ & Flujo ligado máximo & $0.486 \mathrm{~V} \cdot \mathrm{s}$ \\
\hline$\theta_{u}$ & Angulo desalineamiento total & $45^{\circ}$ \\
\hline$\theta_{a}$ & Angulo alineamiento total & $0^{\circ}$ \\
\hline$\beta_{s}$ & Longitud del arco de polo estator & $45^{\circ}$. \\
\hline$\beta_{r}$ & Longitud del arco del polo Rotor & $32^{\circ}$ \\
\hline
\end{tabular}

\section{BIBLIOGRAFIA}

[1] BUJA, Giuseppe; VALLA, Maria. Control Characteristics of the SRM Drives-Part I: Operation in the Linear Region. IEEE Transactions On Industrial Electronics, 1991, Vol. 38, N5, p. $313-322$.

[2] BUJA, Giuseppe; VALLA, Maria. Control Characteristics of the SRM Drives-Part II: Operation in the Saturated Region. IEEE Transactions On Industrial Electronics, 1994, Vol. N³, p. 316-325.

[3] BUJA, G.; VALLAH, U. Control Characteristics of Switched-Reluctance Motor Drives". Proceedings of the IEEE International Workshop on Intelligence Motion Control, August 1990, Vol. 2, p. 615-682.

[4] HOANG, L.; BRUNELLE, Patrice. A Versatile Nonlinear Swiched Reluctance Motor Model in Simulink using Realistic and Analitical Magnetization Characteristics. IECON 2005 Conference. 2005, p. 1556-1561.
[5] MILLER T J E., Electronic Control of Switched Reluctance Machines. Newnes, 2001.

[7] TORREY, D.; NIU, X.; UNKAUF, E. Analitical modeling of variable reluctance machine magnetization characteristics. IEE-Proceedings on Electric Power Application, 1995,

Vol. $142 \mathrm{~N}^{\circ} 1$, p. 14-22.

[8] WANG, Sen; BURTON, Bruce; HARLEY R. Switched Reluctance Motor Measurements and Simulation Models, IEEE AFRICON 2004. 2004, p. 1161-1168.

Recibido: 20 - Marzo - 2007

Aceptado: 19 - junio - 2007 